\title{
Work related physical activity and risk of a hospital discharge diagnosis of atrial fibrillation or flutter: the Danish Diet, Cancer, and Health Study
}

\author{
L Frost, P Frost, P Vestergaard
}

Occup Environ Med 2005;62:49-53. doi: 10.1136/oem.2004.014266

See end of article for authors' affiliations .....................

Correspondence to: Dr L Frost, Department of Cardiology A, Aarhus University Hospital, DK8000 Aarhus C, Denmark; rlg041fr@as.aaa.dk

Accepted 27 August 2004

\begin{abstract}
Background and Aims: Excessive sporting activities have been associated with risk of atrial fibrillation. To study if work related physical activity also confers risk of atrial fibrillation or flutter, the association between work related physical strain and the risk of a hospital discharge diagnosis (inpatient as well as outpatient) of atrial fibrillation or flutter was examined.

Methods: A population based prospective cohort study was conducted from December 1993 to December 2001 among 19593 men and 18807 women with a mean age at baseline of 56 years (range 50-65 years) in the Danish Diet, Cancer, and Health Study. The physical strain during working hours was categorised as sedentary, light, or heavy, and analysed using proportional hazard models. Subjects were followed up in the Danish National Registry of Patients and in the Danish Civil Registration System.

Results: During follow up (mean 5.7 years) a hospital discharge diagnosis of atrial fibrillation or flutter occurred in 305 men and 113 women. When using the risk of atrial fibrillation or flutter associated with sedentary work at a sitting position as a reference, no excess risk (unadjusted as well as adjusted) was found of atrial fibrillation or flutter associated with sedentary work in a standing position, light workload, or heavy workload in men or women.

Conclusion: No evidence was found of an association between physical activities during working hours and risk of a hospital discharge diagnosis of atrial fibrillation or flutter for men and women in the age group of 50-65 years.
\end{abstract}

$\mathrm{E}$ xcessive physical activities such as long lasting (years) endurance sport have been associated with risk of atrial fibrillation in men. ${ }^{1-5}$ The risk of lone atrial fibrillation among endurance athletes has been reported to be in the range $0-5.3 \%,{ }^{14}$ and a case-control study found an odds ratio of 5.5 (95\% CI 1.3 to 24.4) of lone atrial fibrillation among vigorously exercising middle aged men. ${ }^{4}$ Knowledge about the potential risk of atrial fibrillation associated with daily life activities such as physical strain during working hours is, as far as we know, non-existent. We therefore examined the association between work related physical activities and the risk of a hospital discharge diagnosis of atrial fibrillation or flutter among men and women participating in the Danish Diet, Cancer, and Health Study.

\section{METHODS}

\section{Design and study population}

The Danish Diet, Cancer, and Health study is a prospective cohort study with the primary aim of studying the role of diet in cancer risk, but with a potential for studying other diseases as well. The study design has been described in detail elsewhere. ${ }^{6}{ }^{7}$

From December 1993 to May 1997, 80996 men and 79729 women aged 50-64 years were invited to participate in the study; 27177 men and 29876 women accepted the invitation. Eligible cohort members were born in Denmark, living in the Copenhagen and Aarhus areas, and had no previous cancer diagnosis in the Danish Cancer Registry. The baseline data were linked to the Danish Cancer Registry and other population based registries, including the Danish National Registry of Patients, and the Danish Civil Registration System, using the civil registry number, which is a unique number given to everyone living with an address in Denmark since 1968. The Civil Registration System has electronic records of all changes in vital status for the Danish population since 1968, including change of address, date of emigration, and date of death. The Danish National Registry of Patients was established in 1977, and records $99.4 \%$ of all discharges from non-psychiatric hospitals in Denmark. ${ }^{8}$ The data include the civil registry number, dates of admission and discharge, surgical procedures performed, and up to 20 discharge diagnoses per discharge, classified until 1993 according to the Danish version of the International Classification of Diseases, 8th revision (ICD-8), and thereafter according to the national version of ICD-10. The physician who discharged the patient coded all discharge diagnoses. In order to study incident cases of atrial fibrillation, and to reduce confounding, we excluded participants who had been hospitalised before baseline with endocrine diseases or cardiovascular diseases other than hypertension, recorded in the National Registry of Patients, ICD-8 codes 240-279, 390-398, and 410-458, and ICD-10 codes E00-E90, I00-I09, and I16-I99. We did not exclude patients with hypertension before or at baseline for several reasons. First, the diagnostic criteria for hypertension have changed over the past decades. Second, the validity of a diagnosis of hypertension in the Danish National Register of Patients is low. ${ }^{9}$ Third, if we used a definition of hypertension as a systolic blood pressure greater than $140 \mathrm{~mm} \mathrm{Hg}$ at baseline, we would exclude more than $50 \%$ of subjects from the cohort. ${ }^{6}$ Finally, we did not a priori feel that the relation between blood pressure and risk of atrial fibrillation included a threshold function allowing us to exclude any subjects from the cohort due to a specific level of blood pressure.

The Danish Diet, Cancer, and Health Study and the present study were approved by the Regional Ethics Committees in Copenhagen and Aarhus, and by The Danish Data Protection Agency. 
Main messages

- The association of atrial fibrillation and atrial flutter with work related physical strain has not previously been examined.

- In a large cohort study, no excess risk of atrial fibrillation or flutter associated with work related physical activities for men and women in the age group 50-65 years was found.

\section{Baseline data}

Height, weight, systolic and diastolic blood pressure, and total serum cholesterol were measured at baseline. Body weight was measured by the use of a Soehnle digital scale weight, and recorded to the nearest 100 grams. Systolic and diastolic blood pressure were measured by an automatic blood pressure measurement device (Takeda UH 751). Nonfasting total serum cholesterol was measured according to national guidelines. ${ }^{10}$

All participants filled in a questionnaire about medical diseases including myocardial infarction, angina, stroke, hypertension, hypercholesterolaemia, and diabetes, and drug treatment of those conditions. Subjects who reported that they had ischaemic heart disease, stroke, diabetes, and/or were medicated for those conditions were excluded from the present study.

The participants also completed a questionnaire about smoking habits, alcohol intake, health, and duration of education.

\section{Ascertainment of physical activity}

The study participants completed a questionnaire about physical activities during working hours and during leisure time. ${ }^{11}$ Information on physical activities during working hours was obtained by asking: "How was your physical activity at work during the last year?" The participants had to chose between five different possibilities: (1) Sedentary work in the sitting position (mainly sedentary work in a sitting position, not much walking around). (2) Sedentary work in the standing position (mainly sedentary work in a standing or walking position, no carrying of heavy objects). (3) Light physical work (walking a lot, including stairs; carrying light objects). (4) Heavy physical workload (carrying heavy objects; exhausting workload such as manual digging, heavy construction, and working in the forest). (5) Has not been working during the last year. The study participants were also asked how many hours they spent in sporting activities per week during the summer and during the winter. The mean duration of sporting activities during summer and winter was used in the statistical analyses.

\section{Atrial fibrillation and atrial flutter}

The general health and hospital care systems in Denmark are no charge and non-profit systems that are financed through taxes. During the study period there was a very limited capacity in private specialist practices and in private hospitals, so the vast majority of patients with atrial fibrillation were seen in public hospital beds or in outpatient clinics affiliated with public hospitals. We identified probable cases of atrial fibrillation or flutter within the cohort in the Danish National Registry of Patients-that is, cases with the discharge diagnoses 427.93, 427.94, and I48 to 31 December 2001. From 1 January 1995, patients who were only seen in an outpatient hospital clinic were also coded into the Danish National Registry of Patients. A change from the International Classification of Disease, 8th revision to the
Policy implications

- Occupational exposure to a sedentary, mild, or heavy workload does not seem to be associated with risk of atrial fibrillation or flutter.

International Classification of Disease, 10th revision occurred in Denmark in 1994. Atrial fibrillation and atrial flutter were coded separately in the International Classification of Disease, 8th revision (codes 427.93 and 427.94), but in the International Classification of Disease, 10th revision, atrial fibrillation and flutter have the same International Classification of Disease code (I48).

A single reviewer (LF), using a standardised form, reviewed the medical records of the subset of study participants living in the county of Aarhus with an incident diagnosis of atrial fibrillation or flutter recorded in the Danish National Registry of Patients to the end of December 1999. Of 116 subjects with an incident diagnosis of atrial fibrillation or flutter, an electrocardiogram, a printout from telemetry, or a printout from a Holter recording could verify atrial fibrillation or atrial flutter in 112 individuals. Thus, 112 of 116, corresponding to $97 \%$ of the diagnoses, could be verified. Among the 112 subjects with a verified diagnosis, 103 subjects (92\%) had atrial fibrillation, three (3\%) had both atrial fibrillation and atrial flutter, and six $(5 \%)$ had atrial flutter.

\section{Follow up}

The study participants were followed up in the National Registry of Patients and in the Central Person Registry. Linking was done by use of the civil registry number. Follow up for atrial fibrillation or flutter began on the date of visit to one of the study centres, and ended on the date of an event or a censoring (that is, a diagnosis of atrial fibrillation or flutter, death, emigration, or 31 December 2001, whichever came first).

\section{Statistical analyses}

We used piecewise linear regression to examine the relation between a continuous variable and the hazard of atrial fibrillation or flutter. ${ }^{12}$ We kept a continuous variable as continuous in the Cox regression model, when appropriate, according to these analyses.

We computed a multivariate Cox regression model by an initial forced entry of known risk factors for atrial fibrillation, namely age, systolic pressure, treatment for hypertension, together with information on body height and body mass index, followed by selection of the other variables of interest. Thereafter, we confirmed our model by doing a Cox regression analysis with backward elimination of variables. The relevance of a variable in the model was further assessed by the change-in-estimate method. ${ }^{13}$

The variables included in our final models were the categorised exposure information on physical activity during working hours, duration of sporting activities per week, age (years), body height $(\mathrm{cm})$, body mass index $\left(\mathrm{kg} / \mathrm{m}^{2}\right)$, systolic blood pressure $(\mathrm{mm} \mathrm{Hg})$, treatment for hypertension (no, yes), total serum cholesterol $>6 \mathrm{mmol} / \mathrm{l}$ (no, yes), and alcohol consumption (units/day). Thereafter, we assessed further potential confounders: smoking (never, former, current), and length of education after elementary school was included with four categories ( 0 years, $<3$ years, 3-4 years, and $>4$ years) to evaluate the potentials for a change in the estimate of the hazards for atrial fibrillation or flutter associated with physical activity. 
Table 1 Characteristics of men and women at baseline according to sex specific physical activity during working hours; the Danish Diet, Cancer, and Health Study

\begin{tabular}{|c|c|c|c|c|c|}
\hline Characteristic & $\begin{array}{l}\text { Sedentary } \\
\text { (predominantly sitting } \\
\text { position) }\end{array}$ & $\begin{array}{l}\text { Sedentary } \\
\text { (predominantly } \\
\text { standing position) }\end{array}$ & $\begin{array}{l}\text { Light } \\
\text { workload }\end{array}$ & $\begin{array}{l}\text { Heavy } \\
\text { workload }\end{array}$ & $\begin{array}{l}\text { p value for } \\
\text { correlation* }\end{array}$ \\
\hline \multicolumn{6}{|l|}{ Men } \\
\hline Number of subjects & 9240 & 3875 & 4618 & 1860 & \\
\hline Age (years) & $55.2(4)$ & $55.4(4)$ & $55.5(4)$ & $55.2(4)$ & $<0.01$ \\
\hline Body height $(\mathrm{cm})$ & $178.0(6)$ & $176.6(6)$ & $176.0(6)$ & $175.3(6)$ & $<0.01$ \\
\hline Body mass index $\left(\mathrm{kg} / \mathrm{m}^{2}\right)$ & $26.3(3.4)$ & $26.2(3.4)$ & $26.5(3.4)$ & $27.3(3.7)$ & $<0.01$ \\
\hline \multicolumn{6}{|l|}{ Smoking $\dagger$} \\
\hline Never (\%) & 29 & 28 & 28 & 24 & \\
\hline Former (\%) & 37 & 32 & 32 & 30 & \\
\hline Current (\%) & 34 & 40 & 40 & 46 & $<0.01$ \\
\hline Alcohol consumption (g/day) & $28(23)$ & $28(25)$ & $28(26)$ & 32 (29) & $<0.05$ \\
\hline Systolic blood pressure (mm Hg) & $140(19)$ & $141(19 \pm$ & $142(19)$ & $143(19)$ & $<0.01$ \\
\hline Treatment for hypertension (\%) & 9.2 & 7.7 & 8.1 & 7.7 & $<0.01$ \\
\hline Total serum cholesterol $>6 \mathrm{mmol} / \mathrm{l}(\%)$ & 44 & 46 & 45 & 47 & $<0.05$ \\
\hline Duration of sporting activities, hours per week & $1.5(2.2)$ & $1.3(2.1)$ & $1.4(2.3)$ & $1.1(2.3)$ & $<0.01$ \\
\hline \multicolumn{6}{|l|}{ Length of education after elementary schoolt } \\
\hline 0 years $(\%)$ & 4 & 8 & 13 & 20 & \\
\hline$<3$ years $(\%)$ & 13 & 14 & 17 & 15 & \\
\hline $3-4$ years $(\%)$ & 35 & 46 & 47 & 49 & \\
\hline$>4$ years $(\%)$ & 48 & 33 & 24 & 16 & $<0.01$ \\
\hline \multicolumn{6}{|l|}{ Women } \\
\hline Number of subjects & 8605 & 4572 & 5232 & 398 & \\
\hline Age (years) & $55.0(4)$ & $55.6(4)$ & $55.3(4)$ & $55.7(4)$ & $<0.01$ \\
\hline Body height $(\mathrm{cm})$ & $165.0(6)$ & $164.3(6)$ & $163.9(6)$ & $163.8(6)$ & $<0.01$ \\
\hline Body mass index $\left(\mathrm{kg} / \mathrm{m}^{2}\right)$ & $25.2(4.1)$ & $25.1(4.0)$ & $25.4(4.3)$ & $26.1(4.8)$ & $<0.05$ \\
\hline \multicolumn{6}{|l|}{ Smoking $\dagger$} \\
\hline Never (\%) & 47 & 49 & 45 & 36 & \\
\hline Former (\%) & 24 & 23 & 22 & 17 & \\
\hline Current (\%) & 30 & 29 & 33 & 47 & $<0.01$ \\
\hline Alcohol consumption (g/day) & $15(16)$ & $14(15)$ & $13(14)$ & $11(13)$ & $<0.01$ \\
\hline Systolic blood pressure $(\mathrm{mm} \mathrm{Hg})$ & $136(21)$ & $136(21)$ & $136(21)$ & $139(21)$ & 0.13 \\
\hline Treatment for hypertension (\%) & 10.5 & 10.2 & 10.5 & $10.3^{1}$ & 0.81 \\
\hline Total serum cholesterol $>6 \mathrm{mmol} / \mathrm{l}(\%)$ & 50 & 52 & 54 & 57 & $<0.01$ \\
\hline Duration of sporting activities, hours per week & $1.3(1.8)$ & $1.3(1.9)$ & $1.3(1.9)$ & $1.2(2.3)$ & $<0.05$ \\
\hline \multicolumn{6}{|l|}{ Length of education after elementary schoolt } \\
\hline 0 years $(\%)$ & 9 & 20 & 19 & 30 & \\
\hline$<3$ years $(\%)$ & 33 & 26 & 36 & 38 & \\
\hline $3-4$ years $(\%)$ & 41 & 39 & 38 & 25 & \\
\hline$>4$ years $(\%)$ & 17 & 16 & 8 & 6 & $<0.01$ \\
\hline
\end{tabular}

Values are number, mean (SD), or percentage.

*Test for correlation used Spearman correlation analysis. Test results can be significant due to decimals that are not shown in the table.

†Sum of percentages may deviate from 100 due to rounding.

We assessed the possibility of interaction by entering product terms that included the exposures of interest and potential confounders.

The assumption of proportional hazards in the Cox models was evaluated using graphical assessment and found to be appropriate in all models. We calculated 95\% confidence intervals throughout the analyses. We used SPSS statistical software version 11.5 (SPSS Inc., Chicago, IL).

\section{RESULTS}

The cohort included 57053 subjects at baseline. We excluded 9022 subjects who reported that they suffered from, or took medicine for endocrine or cardiovascular diseases (hypertension was not excluded), and/or had a diagnosis of endocrine or cardiovascular disease (hypertension was not excluded) in the Danish National Registry of Patients before or at the date of the baseline. An additional 42 subjects were excluded due to a missing questionnaire. Furthermore, 2881 men and 6478 women were excluded as they reported that they did not work. In 75 men and 155 women there was no information on work related physical activities.

Thus, the study population included into the cohort consisted of 19593 men with a mean age of 55.3 years and 18807 women with a mean age of 55.2 years. The mean duration of follow up was 5.7 years with a range of $0-8.1$ years. During follow up 305 men and 113 women had an incident diagnosis of atrial fibrillation or flutter in the National Registry of Patients.

Table 1 shows the characteristics of men and women at baseline according to physical activities during working hours. Most men and women were exposed to a sedentary occupation or a light workload. Only a few men and women were exposed to a heavy workload.

The incidence rates of atrial fibrillation did not vary much by category of work related physical activity (table 2 ). In men, the incidence rates per 10000 person-years were 27.7, $26.3,26.8$, and 28.9, among subjects with sedentary work ( sitting position), sedentary work (standing position), light workload, and heavy workload, respectively. In women the corresponding incidence rates were 12.2, 9.0, 8.2, and 12.6 .

Table 3 shows the unadjusted and adjusted hazard ratios of atrial fibrillation or flutter according to category of physical workload during working hours. The hazard of atrial fibrillation or flutter did not change much by workload. The inclusion of information on duration of sport activities did not change the hazard for atrial fibrillation or flutter by much (a relative change of the hazard ratio of 3\% and 6\% was observed in men and women). We did not find any significant effect modification. 
Table 2 Incidence rates of atrial fibrillation or flutter according to sex specific physical activity during working hours; the Danish Diet, Cancer, and Health Study

\begin{tabular}{|c|c|c|c|c|}
\hline & $\begin{array}{l}\text { Sedentary } \\
\text { (predominantly sitting } \\
\text { position) }\end{array}$ & $\begin{array}{l}\text { Sedentary (predominantly } \\
\text { standing position) }\end{array}$ & Light workload & Heavy workload \\
\hline \multicolumn{5}{|l|}{ Men } \\
\hline Mean duration of follow up, days & 2071 & 2077 & 2097 & 2105 \\
\hline Total number of person-years of follow up & 52387 & 22039 & 26511 & 10719 \\
\hline Number of subjects with atrial fibrillation or flutter & 145 & 58 & 71 & 31 \\
\hline Incidence rate per 10000 person-years & 27.7 & 26.3 & 26.8 & 28.9 \\
\hline \multicolumn{5}{|l|}{ Women } \\
\hline Mean duration of follow up, days & 2123 & 2127 & 2132 & 2194 \\
\hline Total number of person-years of follow up & 50004 & 26624 & 30533 & 2390 \\
\hline Number of subjects with atrial fibrillation or flutter & 61 & 24 & 25 & 3 \\
\hline Incidence rate per 10000 person-years & 12.2 & 9.0 & 8.2 & 12.6 \\
\hline
\end{tabular}

\section{DISCUSSION}

We did not find any association between working hours physical activities and risk of atrial fibrillation or flutter. This indicates that the association may be so weak that we could not detect it in our study. It may also be that publication bias may have lead to an exaggeration of the risk of atrial fibrillation associated with endurance sporting activities reported in the medical literature.

The main strengths of our study are the large number of cases with atrial fibrillation, the detailed information on potential confounding factors, the complete follow up through nationwide, population based registries, which limit selection and surveillance bias, and the standardised assessment of a sample of the registered outcomes. We used restriction in applying admissibility criteria to reduce confounding and increase validity. ${ }^{14}$

We did not have any information on the intensity or the metabolic equivalency of the self reported physical activities during working hours; this might have caused misclassification of exposure, which could introduce bias in any direction.

Some subjects may have retired from work, before the development of atrial fibrillation, but exclusion of subjects older than 60 years did not change any estimate by much. We used the mean duration of the reported sporting activities during summer and winter. However, reanalysis of our data, with use of specific summer and winter activities, did not change any major conclusions. We could not analyse the potential for risk associated with sharp fluctuations in activity levels, as this question may best be answered by use of the case-crossover design. ${ }^{15}$

Atrial fibrillation may be associated with more symptoms in subjects who are physically active. If this is the case, we are left with a bias towards more atrial fibrillation or flutter being diagnosed in subjects with a high level of physical activity, and then we have underestimated the hazard of atrial fibrillation among subjects with a sedentary lifestyle. However, we find it unlikely that follow up should be biased by category of work related physical activity.

We were only able to include atrial fibrillation or flutter that was symptomatic and led to clinical investigation in an outpatient hospital clinic or a hospital bed. However, given the age profile of our study cohort, it is very likely that patients with clinical symptoms of atrial fibrillation were referred for further evaluation; this seems highly possible, as the incidence rates for atrial fibrillation or atrial flutter in men and women in our study are in accordance with the incidence rates for atrial fibrillation in the same age interval reported from the Framingham Heart Study, ${ }^{16}$ The Manitoba Follow-up Study, ${ }^{17}$ and the Renfrew/Paisley study. ${ }^{18}$

We could not differentiate atrial fibrillation from atrial flutter, as atrial fibrillation and atrial flutter had the same ICD code. However, from the evaluation of hospital files of a sample of cases participating in Danish Diet, Cancer, and Health Study, and recorded in the Danish National Registry of Patients with an incident diagnosis of atrial fibrillation or flutter, we know that approximately $5 \%$ of the recorded cases had atrial flutter. Given the low proportion of patients with atrial flutter, the potentials of bias by inclusion of atrial flutter would be modest.

We had limited statistical power, and we cannot, due to the observational design, exclude the possibility of unmeasured bias, and uncontrolled confounding. However, we find it reassuring that daily life activity such as work related physical activities can be done without risk of adverse events in the form of atrial fibrillation or flutter.

Table 3 Risk of atrial fibrillation or flutter according to sex specific physical activity during working hours; the Danish Diet, Cancer, and Health Study

\begin{tabular}{lllll}
\hline & $\begin{array}{l}\text { Sedentary* } \\
\text { (predominantly sitting } \\
\text { position) }\end{array}$ & $\begin{array}{l}\text { Sedentary (predominantly } \\
\text { standing position) }\end{array}$ & Light workload & Heavy workload \\
\hline Men & 1.00 & $0.95(0.70$ to 1.29$)$ & $0.97(0.73$ to 1.28$)$ & $1.04(0.71$ to 1.54$)$ \\
Unadjusted hazard rate ratio $(95 \% \mathrm{Cl})$ & 1.00 & $1.01(0.74$ to 1.38$)$ & $0.97(0.72$ to 1.32$)$ \\
Adjusted hazard rate ratiot $(95 \% \mathrm{Cl})$ & $1.09(0.72$ to 1.64$)$ \\
Women & 1.00 & $0.74(0.46$ to 1.18$)$ & $0.67(0.42$ to 1.07$)$ & $1.02(0.32$ to 3.24$)$ \\
Unadjusted hazard rate ratio $(95 \% \mathrm{Cl})$ & 1.00 & $0.74(0.45$ to 1.22$)$ & $0.73(0.45$ to 1.19$)$ & $1.15(0.36$ to 3.70$)$ \\
\hline Adjusted hazard rate ratiot $(95 \% \mathrm{Cl})$ & &
\end{tabular}

*Reference category.

†Adjusted for age, body height, body mass index, smoking, consumption of alcohol, systolic blood pressure, treatment for hypertension, total serum cholesterol, duration of sporting activities, and level of education. 


\section{ACKNOWLEDGEMENTS}

Lars Frost received a senior research fellowship from The Danish Medical Research Council (grant number 22-02-0582). Katja Bool, programmer, and Jytte Fogh Larsen, secretary, both at The Danish Cancer Society, are thanked for assisting with data collection. Anne Tjønneland, MD, PhD and Kim Overvad, MD, PhD are thanked for access to data. Lone Juul Hansen, statistician, UNI-C, The Danish IT Centre for Education and Research assisted with data management and statistical analyses.

\section{Authors' affiliations}

L Frost, Department of Cardiology A, Aarhus University Hospital, Denmark

P Frost, Department of Occupational Medicine, Aarhus University Hospital, Denmark

P Vestergaard, Department of Endocrinology C, Aarhus University Hospital, Denmark

Potential conflict of interest: Lars Frost has received funding/grant support for research projects from Boehringer-Ingelheim, Cardiome Pharma, The Danish Heart Foundation, The Danish Medical Research Council, The Danish Society of Nephrology, The Hørslev Foundation, Laerdahls Foundation for Acute Medicine, Lægekredsforeningens Forskningsfond, Merck, Nycomed, and Pfizer, and he serves/served as a consultant for AstraZeneca and Pfizer.

\section{REFERENCES}

1 Zehender M, Meinertz T, Keul J, et al. ECG variants and cardiac arrhythmias in athletes: clinical relevance and prognostic importance. Am Heart $J$ 1990;119:1378-91.

2 Sivertssen E, Benestad AM, Rykke E, et al. [ls top level athletic performance dangerous? A 25-year follow-up study of 24 elite cross-country skiers]. Tidsskr Nor Laegeforen 1994; 114:3300-4

3 Furlanello F, Bertoldi A, Dallago $M$, et al. Atrial fibrillation in elite athletes. J Cardiovasc Electrophysiol 1998;9:S63-8.
4 Karjalainen J, Kujala UM, Kaprio J, et al. Lone atrial fibrillation in vigorously exercising middle aged men: case-control study. BMJ 1998;316:1784-5.

5 Mont L, Sambola A, Brugada J, et al. Long-lasting sport practice and lone atrial fibrillation. Eur Heart J 2002;23:477-82.

6 Tjonneland AM, Overvad OK. [Diet, cancer and health-a population study and establishment of a biological bank in Denmark]. Ugeskr Laeger 2000;162:350-4.

7 Riboli E, Hunt KJ, Slimani N, et al. European Prospective Investigation into Cancer and Nutrition (EPIC): study populations and data collection. Public Health Nutr 2002;5:11113-24.

8 Andersen TF, Madsen M, Jorgensen J, et al. The Danish National Hospital Register. A valuable source of data for modern health sciences. Dan Med Bull 1999;46:263-8.

9 Nielsen HW, Tuchsen F, Jensen MV. [Validity of the diagnosis "essential hypertension" in the National Patient Registry]. Ugeskr Laeger 1996;158:163-7.

10 Dyerberg J, Stender S, Andersen GE, et al. [Recommendations for clinicalchemical departments: lipid-lipoprotein analysis]. Ugeskr Laeger 1990;152:1434-7.

11 Haftenberger M, Schuit AJ, Tormo MJ, et al. Physical activity of subjects aged 50-64 years involved in the European Prospective Investigation into Cancer and Nutrition (EPIC). Public Health Nutr 2002;5:1163-76.

12 Thomsen BL. A note on the modelling of continuous covariates in Cox's regression model. Research Report 88/5. University of Copenhagen: Statistical Research Unit, 1988.

13 Greenland S. Modeling and variable selection in epidemiologic analysis. Am J Public Health 1989;79:340-9.

14 Rothman KJ. Epidemiology: an introduction. New York: Oxford University Press, 2002.

15 Maclure M, Mittleman MA. Should we use a case-crossover design? Annu Rev Public Health 2000;21:193-221.

16 Benjamin EJ, Levy D, Vaziri SM, et al. Independent risk factors for atrial fibrillation in a population-based cohort. The Framingham Heart Study. JAMA 1994;271:840-4.

17 Krahn AD, Manfreda J, Tate RB, et al. The natural history of atrial fibrillation: incidence, risk factors, and prognosis in the Manitoba Follow-Up Study. Am J Med 1995;98:476-84.

18 Stewart S, Hart CL, Hole DJ, et al. Population prevalence, incidence, and predictors of atrial fibrillation in the Renfrew/Paisley study. Heart 2001;86:516-21. 